\title{
The Coexistence of Low-Grade Mucinous Neoplasms of the Appendix and Appendiceal Diverticula: A Possible Role in the Pathogenesis of Pseudomyxoma Peritonei
}

Laura W. Lamps, M.D., George F. Gray, Jr., M.D., Bradley R. Dilday, M.D., Mary Kay Washington, M.D., Ph.D.

Departments of Pathology, University of Arkansas for Medical Sciences (LWL, BRD), Little Rock, Arkansas, and Vanderbilt University Medical Center (GFG, MKW), Nashville, Tennessee

We examined 38 appendectomies with diagnoses of mucocele, diverticulum, or adenoma to study the coincidence of appendiceal diverticula and appendiceal low-grade mucinous neoplasms and to examine the possible role of diverticula in the pathogenesis of pseudomyxoma peritonei. Invasive adenocarcinomas and retention cysts were excluded (six cases). Cases were classified as adenomas or mucinous tumors of unknown malignant potential, with or without diverticula. Medical records were reviewed for multiple parameters, including presenting symptoms, presence of pseudomyxoma peritonei, and presence of associated malignancies. Binomial statistics were used to calculate the probability that the observed prevalence of low-grade mucinous neoplasms and diverticula together was significantly different from the expected prevalence of diverticula or low-grade mucinous neoplasms alone, using historical controls from the literature.

Twenty-five percent of the total cases ( 8 of 32) contained both a low-grade mucinous neoplasm ( 7 cystadenomas and 1 mucinous tumor of unknown malignant potential) and a diverticulum. Thus, 8 of 19 low-grade mucinous neoplasms $(42 \%)$ were associated with diverticula. Of the appendices with both low-grade mucinous neoplasms and diverticula, three contained dissecting acellular mucin within the appendiceal wall, four showed diverticular perforation, and one had pseudomyxoma peritonei associated with the ruptured diverticulum.

Copyright $\odot 2000$ by The United States and Canadian Academy of Pathology, Inc.

VOL. 13, NO. 5, P. 495, 2000 Printed in the U.S.A.

Date of acceptance: November 5, 1999.

This work was presented in part at the annual meeting of the United States and Canadian Academy of Pathology, Orlando, Florida, March 5, 1997.

Address reprint requests to: Laura W. Lamps, M.D., Department of Pathology, University of Arkansas for Medical Sciences, 4301 West Markham Street, Slot 517, Little Rock, AR 72205; e-mail: lampslauraw@exchange.uams.edu; fax: 501-603-1479.
A significant percentage $(P<.001)$ of cases contained low-grade mucinous neoplasms and diverticula together. The case of coexistent low-grade mucinous neoplasm, diverticulum, and pseudomyxoma peritonei suggests that diverticula could play a role in the pathogenesis of pseudomyxoma peritonei. This could occur either by involvement of preexisting diverticula by the neoplasm or by distention of the appendiceal lumen by mucin, leading to increased intraluminal pressure and subsequent diverticulum formation at a weak area in the wall. Either mechanism might allow low-grade mucinous neoplasms to penetrate the appendiceal wall more easily.

KEY WORDS: Appendiceal mucinous tumor, Appendiceal diverticula, Mucinous cystadenoma, Mucinous tumor of unknown malignant potential, Pseudomyxoma peritonei.

Mod Pathol 2000;13(5):495-501

Diverticula of the appendix are uncommon, reportedly present in 0.004 to $2 \%$ of appendectomies (1$3)$. The incidence of these lesions may be underreported because of their small size (often $<.5 \mathrm{~cm}$ ), causing them to be overlooked at gross examination. In addition, they are easily obliterated by superimposed diverticular inflammation, making both gross and microscopic identification difficult. Acquired appendiceal diverticula are approximately 10 times more common than the congenital type (3, 4 ), and the pathogenesis of these acquired lesions is controversial. Theories historically have included a postinflammatory reaction, weakness in the appendiceal wall as a result of the vascular hiatus, and lumenal obstruction (2). Acquired diverticula have been associated with numerous conditions, including appendiceal adenocarcinoma and benign neural lesions $(2,5,6)$; there is also an association with cystic fibrosis (7). 
Low-grade mucin-producing tumors of the appendix, including adenomas and mucinous tumors of unknown malignant potential (MTUMPs), may be associated with pseudomyxoma peritonei (812). Pseudomyxoma peritonei has been defined by the presence of intraperitoneal mucin, either with or without associated mucin-producing epithelium, and often accompanied by fibrosis and granulation tissue $(9-11)$. However, the mechanism by which mucin traverses the appendiceal wall in benign or low-grade neoplasms has never been fully elucidated, especially in cases in which unequivocal invasion of the appendiceal wall is not present (11). The association of appendiceal diverticula and lowgrade epithelial neoplasms has been noted but has not been investigated extensively $(8,13)$. In one large study, approximately $34 \%$ of appendiceal cystadenomas were associated with diverticula (13), but to our knowledge, this association and its implications have not been subsequently examined. There have also been rare reports of pseudomyxoma peritonei associated with appendiceal diverticula alone $(2,5,14,15)$. As the possible associations among diverticula, low-grade appendiceal mucinous tumors, and pseudomyxoma peritonei have not been well characterized, our goal was to explore associations among these lesions and to examine the possible role of appendiceal diverticula in the pathogenesis of pseudomyxoma peritonei.

\section{MATERIALS AND METHODS}

Appendectomy cases diagnosed as mucocele, diverticulum, cystadenoma, or adenoma between 1966 and 1998 were retrieved from the surgical pathology files of Vanderbilt University Medical Center and the University of Arkansas for Medical Sciences. All slides from 38 appendices were subsequently reviewed by at least two pathologists. Cases with an overtly malignant epithelial component, defined by severe nuclear atypia and/or unequivocal invasion of the appendiceal wall, were classified as adenocarcinomas and excluded from the study. Retention cysts, defined as distention of the appendiceal lumen with associated thinning and atrophy of non-neoplastic mucosa, were excluded as well. The remaining 32 cases were then evaluated for multiple histologic features, including the presence or absence of neoplastic epithelium, presence or absence and number of diverticula, fibrous obliteration of the appendiceal tip, acute inflammation, perforation, submucosal and/or lamina propria fibrosis, lymphoid atrophy, and fecaliths. Lesions were then classified as diverticulum alone, adenoma (including cystadenomas) or MTUMP alone, adenoma or MTUMP with associ- ated mucocele, and adenoma or MTUMP with diverticulum using the following definitions (8-11, 16-19):

Diverticulum: herniation of the mucosa and muscularis mucosae through a vascular hiatus in the wall of the appendix.

Adenoma: mucus-filled, often cystic mass lined by low-grade neoplastic tall columnar epithelium in a sessile, usually circumferential growth pattern, without evidence of invasion into the appendiceal wall or high-grade nuclear atypia. Cystadenomas (adenomas with accompanying dilatation of the appendix) were included in this group.

Mucinous tumor of unknown malignant potential: mucus-filled, often cystic tumor lined by lowgrade epithelium similar to that of the adenoma. The epithelium may push deeply into the underlying tissues, but there may not be unequivocal infiltration, single-cell invasion, or a desmoplastic response. Some authors might choose to incorporate adenomas into the MTUMP category. Others separate MTUMP from adenomas on the basis of the presence of mucinous epithelium pushing deeply into the appendiceal wall or the presence of mucin within the wall or outside the appendix (9).

Mucocele: lumenal distention by mucus in association with a mucus-producing lesion, such as a cystadenoma. Mucocele was not used as a specific diagnostic term, as it does not address the pathogenetic mechanism causing the lumenal distention and mucus production.

Available medical records were then reviewed, with attention to the presence or absence of pseudomyxoma peritonei, presenting symptoms, and presence or absence of associated malignancies.

Binomial statistics were used to calculate the probability that the observed coincidence of lowgrade mucinous neoplasms with diverticula was significantly different from the expected prevalence of diverticula or low-grade mucinous neoplasms alone, based on prevalence figures from large series in the literature.

\section{RESULTS}

The 32 cases were classified as shown in Table 1. Twenty-five percent (8 of 32) of the total appendectomy cases contained both an adenoma or an

TABLE 1. Classification of Appendiceal Lesions

\begin{tabular}{lc}
\hline \multicolumn{1}{c}{ Diagnosis } & Percentage of cases \\
\hline Low-grade mucinous neoplasm ${ }^{a}$ alone & $3 \%(1 / 32)$ \\
Low-grade mucinous neoplasm with mucocele & $31 \%(10 / 32)$ \\
Low-grade mucinous neoplasm with diverticulum & $25 \%(8 / 32)$ \\
\hline \multicolumn{2}{c}{ Includes both adenomas and mucinous tumors of unknown malig- } \\
nant potential.
\end{tabular}




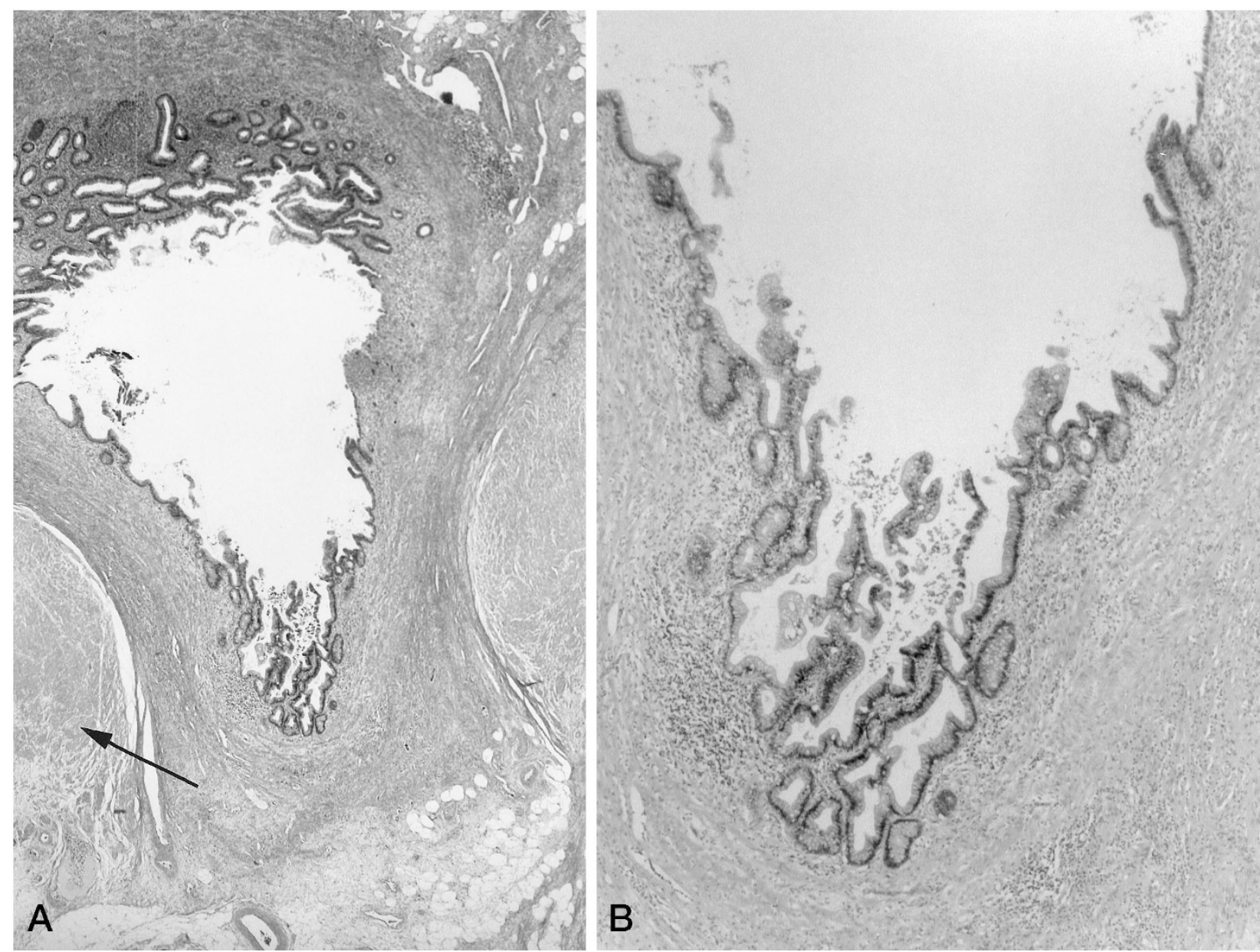

FIGURE 1. A, appendiceal cystadenoma involving diverticulum that penetrates between muscle bundles (arrow) at the vascular hiatus (hematoxylin and eosin, $40 \times$ ). B, higher power view showing adenomatous epithelium present within diverticulum (100 $\times$ ).

MTUMP and a diverticulum; moreover, 8 of the 19 (42\%) low-grade mucinous neoplasms identified in the study contained an associated diverticulum. The diverticula consisted of herniation of the mucosal layer through the appendiceal wall in the area of a vascular hiatus (Fig. 1). In all cases, the neoplastic epithelium was present within the diverticula. Four of these eight cases contained multiple diverticula, and two contained fibrous obliteration of the tip of the appendix. Virtually all cases exhibited lymphoid atrophy and submucosal fibrosis in the wall beneath the adenomatous epithelium. None contained a fecalith. Five had associated acute appendiceal diverticulitis (Fig. 2), and three of these showed perforation of the diverticulum containing the adenomatous epithelium (see below). Three cases showed marked attenuation of the appendiceal wall (1- to 2-mm thickness or less) at the site of the diverticulum (Fig. 3).

Six of the eight cases contained tall columnar, pseudostratified, "ovarian-type" epithelium with basally located nuclei and rare well-defined goblet cells (Fig. 4). One case contained a mixture of this type of epithelium with more villiform epithelium resembling that of a colonic adenoma, and one case consisted almost entirely of this type of villous epithelium (Fig. 5). All neoplastic epithelia were present in an undulating, circumferential growth pattern. No area contained severe nuclear atypia in any of the cases.

Three of the eight cases contained dissecting acellular pools of mucus within the appendiceal wall near an inflamed, perforated diverticulum. Although there was mucus present within the appendiceal wall, none of these three cases had mucus adherent to the serosal surface of the appendix or the mesoappendix. One case, the MTUMP, had associated pseudomyxoma peritonei documented at the time of appendectomy; this patient had a ruptured diverticulum (Fig. 6A) with pools of mucin containing scattered clusters of low-grade epithelium present diffusely throughout the abdominal cavity with associated fibrosis and mixed inflammatory infiltrate, indicating tissue reaction to the mucin (Fig. 6B). The epithelium in the area of the perforated diverticulum had a broad, pushing border but lacked definite invasion and desmoplasia.

In these eight appendices with both coexistent low-grade mucinous neoplasms and diverticula, gross measurements of the appendices ranged from 


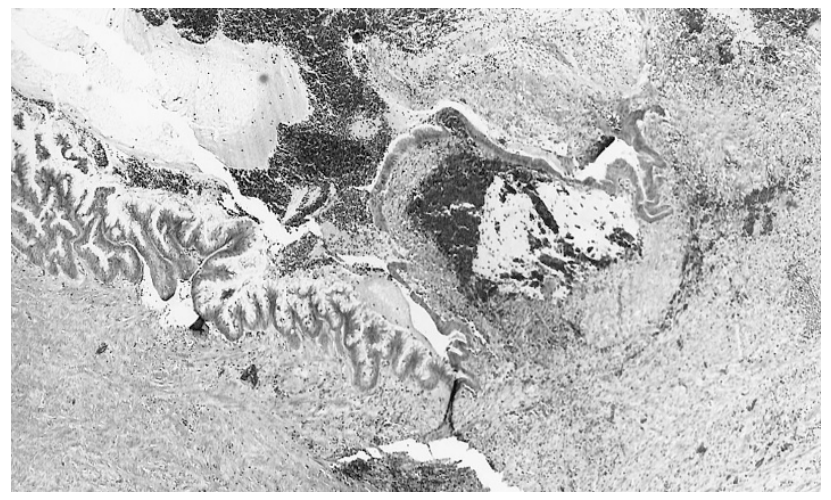

FIGURE 2. Adenomatous epithelium within diverticulum with marked associated acute inflammation (hematoxylin and eosin, $100 \times$ ).

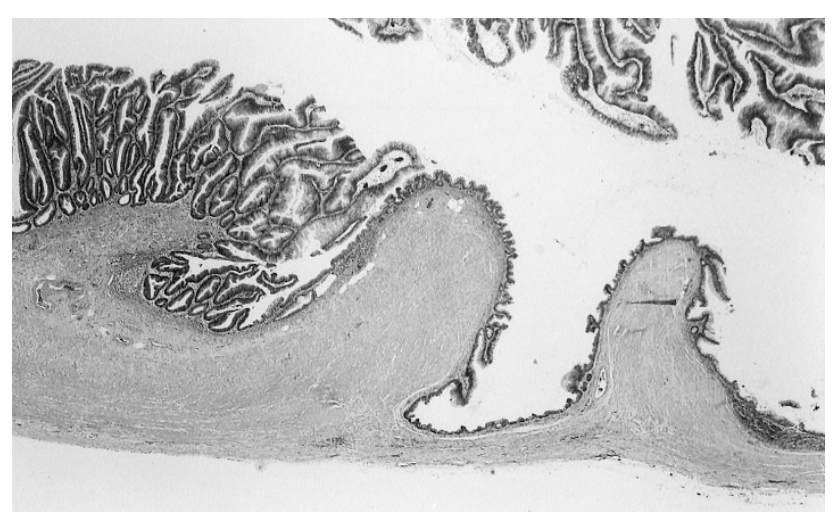

FIGURE 3. Marked attenuation of the appendiceal wall and lymphoid atrophy within a diverticulum involved by a cystadenoma (hematoxylin and eosin, $40 \times$ ).

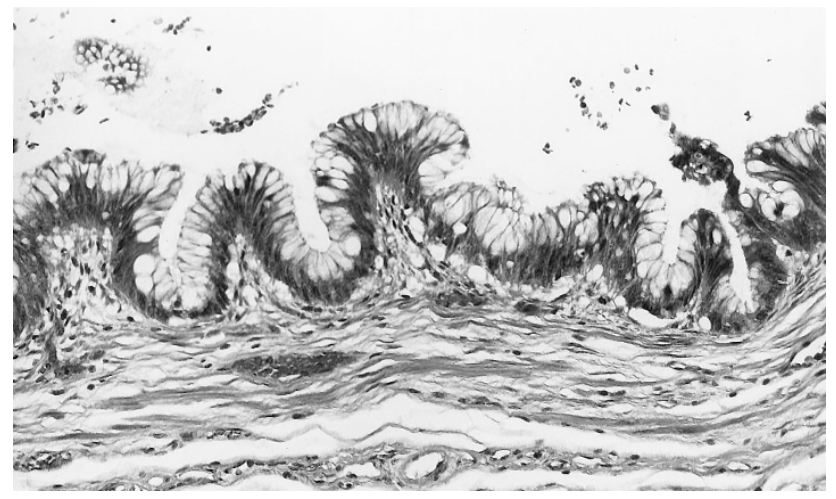

FIGURE 4. Typical epithelium of a cystadenoma with pseudostratified, columnar cells containing elongated, crowded, hyperchromatic nuclei and scattered, well-defined goblet cells (hematoxylin and eosin, $400 \times$ ).

6 to $15 \mathrm{~cm}$ in maximum length and 0.9 to $2 \mathrm{~cm}$ in maximum diameter. Diverticula ranged from 0.2 to $0.8 \mathrm{~cm}$ in maximum dimension. A grossly identifiable mucus-producing lesion with a dilated appendiceal lumen was noted in two cases. In the case with pseudomyxoma peritonei, an intraluminal mass was identified grossly, as well as a perforated diverticulum at the distal end of the appendix; mu-

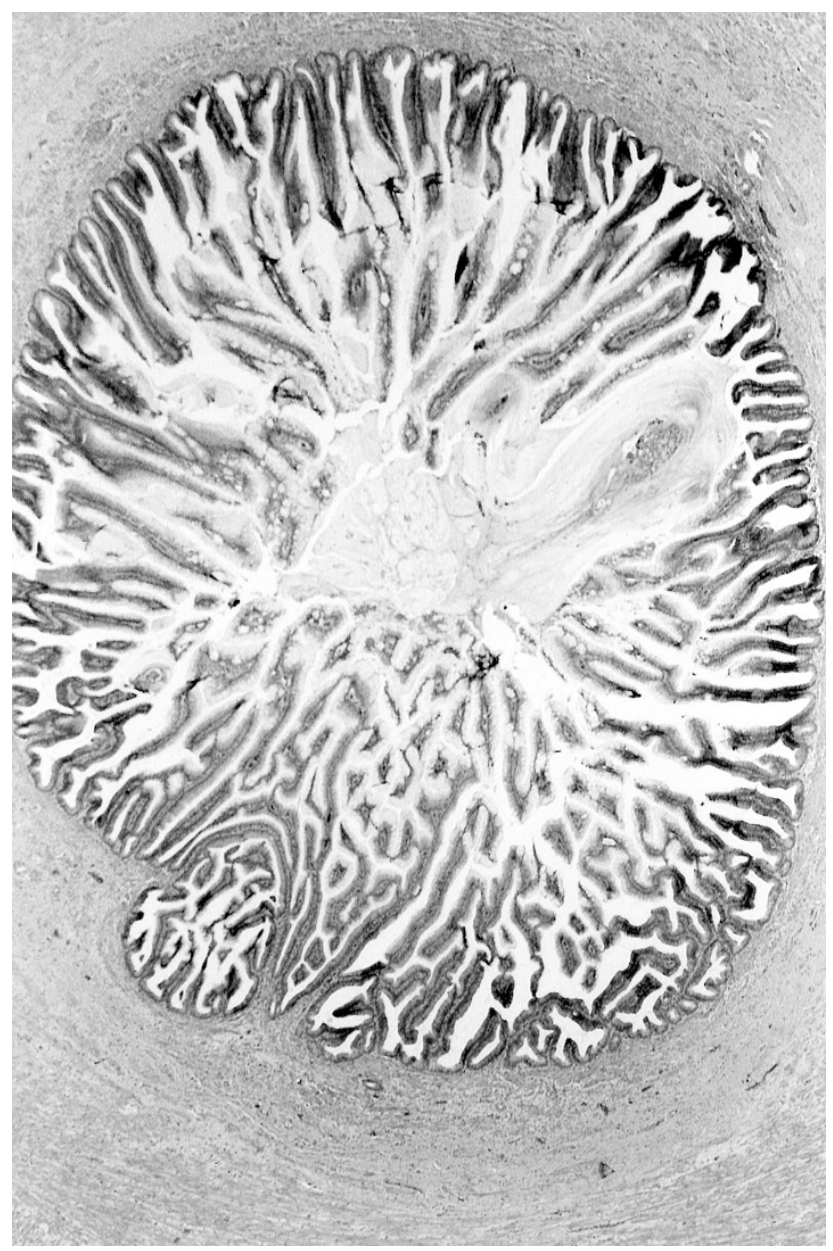

FIGURE 5. Appendiceal adenoma with circumferential, villous epithelium resembling colonic adenoma (hematoxylin and eosin, 40×).

cus was adherent to the serosa of the appendix, gallbladder, and intestines and also involved the omentum.

The patients who had both low-grade mucinous neoplasms and diverticula ranged in age from 41 to 78 years with a mean of 60 years. Five patients were female, two were male, and one was of unknown age and gender (some charts were unavailable for review because of the age of the cases). Indications for surgery were symptoms of acute appendicitis in three patients, symptoms of ulcerative colitis in one patient, an intestinal malignant lymphoma in one patient, a palpable right lower quadrant mass in one patient, right upper quadrant pain and tenderness in one patient, and unknown in the remaining patient. All underwent open appendectomy except for the patient who had lymphoma, who underwent right colectomy, and the patient with ulcerative colitis, who underwent proctocolectomy. None of these patients had associated gastrointestinal or ovarian malignancies. In the patient who had ulcerative colitis, the appendix was not involved by inflammatory bowel disease.

We reviewed the available medical records and found no additional patients who developed 


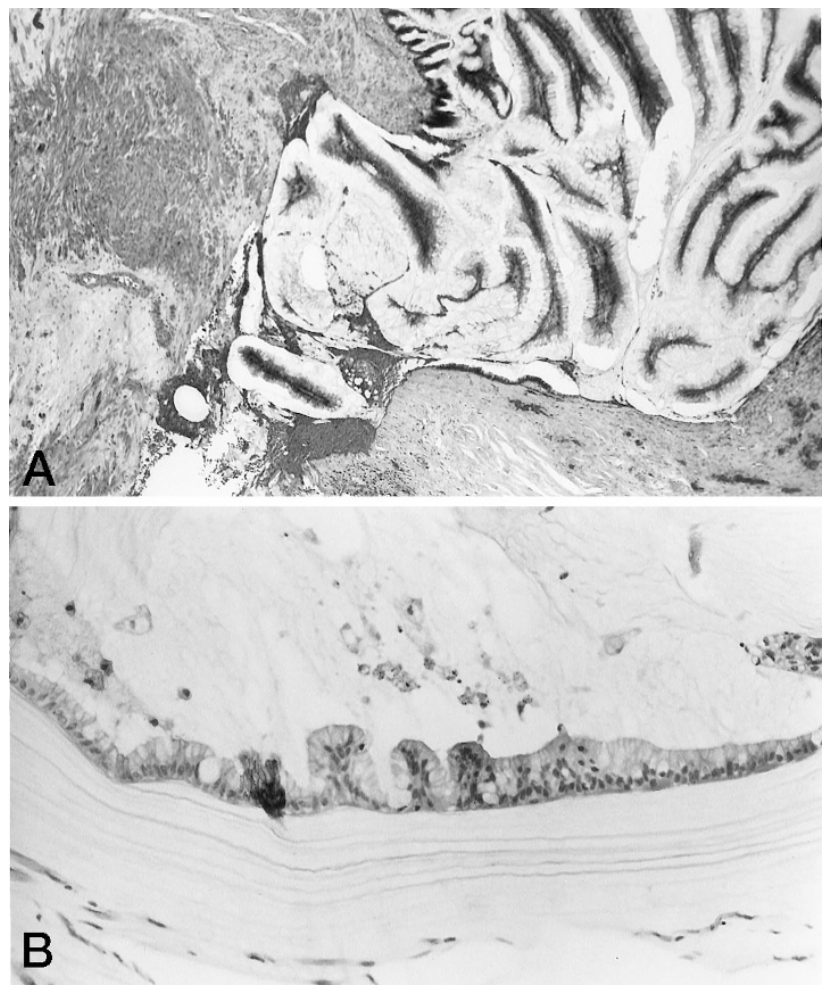

FIGURE 6. A, ruptured appendiceal diverticulum containing adenomatous epithelium (hematoxylin and eosin, $40 \times$ ). B, associated pseudomyxoma peritonei characterized by pools of mucin containing very rare clusters of low-grade epithelium $(200 \times)$.

pseudomyxoma peritonei after their initial appendectomies. Three patients were alive and diseasefree 20 months, 22 months, and 6 years later. The patient who had malignant lymphoma died 2 years later from that disease. The patient who had pseudomyxoma peritonei was alive and free of symptoms 9 months later but with known remaining mucinous peritoneal deposits. Three patients were lost to follow-up.

Using binomial statistics, we calculated the probability that the observed rate of occurrence of lowgrade mucinous neoplasms with diverticula $(42 \%)$ from this study was significantly different from the reported rate of occurrence of these neoplasms in the literature [the reported prevalence of cystadenoma is less than $1 \%$ of resected appendices (20), and that of diverticula is 1 to $2 \%$ of appendectomies (1-3)]. This analysis was done to estimate the likelihood that diverticula occur more frequently in appendices with low-grade mucinous neoplasms, indicating a significant association between these two entities. The $p$ value was less than 0.001 .

\section{DISCUSSION}

Low-grade appendiceal mucinous neoplasms are uncommon; the reported prevalence of cystadenoma is less than $1 \%$ of resected appendices (20). Acquired diverticula are reported in a slightly larger percentage of appendectomies than low-grade mucinous tumors ( 1 to $2 \%$ ) but are still infrequent findings (1-3). The coincidence of these two lesions has not been extensively investigated but was reported in one previous study as approximately $34 \%$ (13).

We found that $42 \%$ of the low-grade mucinous neoplasms in our study had associated diverticula, a prevalence similar to that previously reported (13). In addition, we demonstrated that this association between appendiceal low-grade mucinous neoplasms and diverticula is statistically significant $(P<.001)$.

Seven of the low-grade mucinous neoplasms with associated diverticula were adenomas, and one was a mucinous tumor of unknown malignant potential. Significant associated histologic features included acute inflammation of the diverticula with and without perforation, fibrous obliteration of the tip of the appendix, lymphoid atrophy, submucosal fibrosis, pools of acellular mucin within the wall of the appendix near areas of acute inflammation and perforation, and one case of pseudomyxoma peritonei.

We believe that the coexistence of diverticula and low-grade mucinous neoplasms cannot be explained by chance alone and is important for several reasons. First, neoplastic epithelium extending into a diverticulum, as well as displaced islands of neoplastic epithelium in an inflamed diverticulum, may be mistaken for invasion of the appendiceal wall by a low-grade mucinous tumor. The presence of acellular mucin within the wall of the appendix in association with these low-grade neoplasms may cause a similar diagnostic problem $(8,9,21)$. The lack of atypical cells within dissecting mucin and the absence of desmoplasia may aid in distinguishing mucinous dissection and diverticula containing neoplastic epithelium from a truly invasive neoplasm (9).

Another reason that the coexistence of these rare appendiceal lesions is interesting is their possible role in the pathogenesis of pseudomyxoma peritonei. Pseudomyxoma peritonei is a term that has been used differently by various authors. Clinically, it refers to the jelly-like deposition of mucus on peritoneal surfaces. Microscopically, authors have used this term to mean mucin associated with mucinous epithelia within the implant or acellular mucin deposits; some authors require the presence of associated fibrosis and granulation tissue to make this diagnosis, indicating a reaction to the peritoneal mucin $(8-11,22)$. The mucinous deposits may be confined to the vicinity of the primary tumorcontaining organ or may be widespread and diffuse. The clinical behavior of this lesion seems to depend on the features of the underlying neoplasm $(9,18,22)$. Pseudomyxoma peritonei may accom- 
pany appendiceal lesions that are cytologically benign and that lack unequivocal evidence of invasion $(8,10,11,19)$.

The coincidence of low-grade mucinous neoplasms, diverticula, and pseudomyxoma peritonei that we observed in this study suggests possible roles for appendiceal diverticula in the pathogenesis of pseudomyxoma peritonei. One possibility is that the adenomatous epithelium may grow into a preexisting diverticulum, and the attenuated, often inflamed wall of the diverticulum may provide an easy route of egress for these otherwise benign mucinous tumors. An alternative theory is that the low-grade neoplasm produces mucin that distends the appendiceal lumen and increases intramural pressure. This increase in intramural pressure widens the space where the perforating vessels traverse the muscularis propria, allowing neoplastic epithelium and accompanying mucin to herniate through these weak areas in the appendiceal wall in the form of a diverticulum. The statistically significant association between low-grade mucinous tumors and diverticula shown in this study suggests that diverticula are more commonly present in appendices that contain low-grade mucinous tumors and supports this pathogenetic mechanism. In addition, appendiceal diverticula have been reported in association with other diseases that cause increased mucous production, lumenal obstruction, and thus increased intramural pressure, such as adenocarcinomas and cystic fibrosis (5-7).

It is not our contention that this is a proven or sole mechanism for the development of pseudomyxoma peritonei. However, our case that contained both an MTUMP and a diverticulum, with associated pseudomyxoma peritonei, supports the role that this combination of lesions may play in the pathogenesis of pseudomyxoma peritonei. In this case, the presence of the low-grade neoplasm within the diverticulum, which in turn ruptured because of the presence of the distending mucin and resulted in pseudomyxoma peritonei, is evidence that diverticula may provide an easy route through the appendiceal wall for low-grade mucinous neoplasms.

Some authors have also suggested that the presence of acellular mucin in the wall of the appendix associated with low-grade mucinous neoplasms may represent the stage just before appendiceal rupture with subsequent spillage of mucin and pseudomyxoma peritonei (17). The presence of acellular mucin within the walls of the appendices near areas of diverticular inflammation in three of our cases is additional evidence that mucin production leads to increased intraluminal pressure, which in turn forces mucin to dissect into the wall.
In summary, appendiceal diverticula and lowgrade mucinous neoplasms are unusual appendiceal lesions that coexist more frequently than can be explained by chance alone. These lesions are of importance to the surgical pathologist in that they may be mistaken for an invasive mucinous neoplasm, as well as play a possible role in the pathogenesis of pseudomyxoma peritonei. A careful gross and microscopic search for coexisting diverticula is therefore warranted for patients who have benign or low-grade appendiceal mucinous neoplasms.

Acknowledgments: The authors gratefully acknowledge Dr. Martin Hauer-Jensen, M.D., Ph.D., for assistance with statistical analysis and Dr. Rodger C. Haggitt for his helpful review of the manuscript.

\section{REFERENCES}

1. Payan HM. Diverticular disease of the appendix. Dis Col Rect 1977;20:473-6.

2. Trollope ML, Lindenauer SM. Diverticulosis of the appendix: a collective review. Dis Col Rect 1974;17:200-18.

3. Lipton S, Estrin J, Glasser I. Diverticular disease of the appendix. Surg Gynecol Obstet 1989;168:13-6.

4. Buffo GC, Clair MR, Bonheim P. Diverticulosis of the vermiform appendix. Gastrointest Radiol 1986;11(1):108-9.

5. Wilkie DPD. Carcinoma of the appendix causing diverticula of the appendix and acute appendicular obstruction. Br J Surg 1921;8:392-6.

6. Dordick JR. Diverticula of the vermiform appendix associated with an overgrowth of nerve tissue and partial mucocele. Arch Pathol 1939;27:735-41.

7. George DH. Diverticulosis of the vermiform appendix in patients with cystic fibrosis. Hum Pathol 1987;18(1):75-9.

8. Appelman HD. Epithelial neoplasia of the appendix. In: Norris HT, editor. Pathology of the colon, small intestine, and anus. 2nd ed. New York: Churchill Livingstone; 1991. p. 263-303.

9. Carr NJ, Sobin LH. Unusual tumors of the appendix and pseudomyxoma peritonei. Semin Diagn Pathol 1996;13: 314-25.

10. Young RH, Gilks CB, Scully RE. Mucinous tumors of the appendix associated with mucinous tumors of the ovary and pseudomyxoma peritonei. Am J Surg Pathol 1991;15(5): 415-29.

11. Ronnett BM, Zahn CM, Kurman RJ, Kass ME, Sugarbaker PH, Shmookler BM. Disseminated peritoneal adenomucinosis and peritoneal mucinous carcinomatosis. Am J Surg Pathol 1995;19:1390-408.

12. Landen S, Bertrand C, Maddern GJ, Herman D, Pourbaix A, de Neve A, et al. Appendiceal mucoceles and pseudomyxoma peritonei. Surg Gynecol Obstet 1992;175:401-4.

13. Qizilbash AH. Mucoceles of the appendix. Arch Pathol 1975; 99:548-55.

14. Dialti G. Pseudomixoma del peritoneo di origine appendicolare in ernia dell'appendice. Clin Chir 1914;22:1729-78.

15. Lohr W. Zur frage des wurmforsatzdivertikels und seiner Folgezustande, insbesondere des Pseudomyxoma peritonei. Dtsch Z Chir 1922;171:30-56.

16. Isaacs KL, Warshauer DM. Mucocele of the appendix: computed tomographic, endoscopic, and pathologic correlation. Am J Gastroenterol 1992;87:787-9.

17. Wolff M, Ahmed N. Epithelial neoplasms of the vermiform 
appendix (exclusive of carcinoid). Cancer 1976;37:2511-22.

18. Higa E, Rosai J, Pizzimbono CA, Wise L. Mucosal hyperplasia, mucinous cystadenoma, and mucinous cystadenocarcinoma of the appendix: a re-evaluation of appendiceal "mucocele." Cancer 1973;32:1525-41.

19. Wackym PA, Gray GF. Tumors of the appendix: neoplastic and nonneoplastic mucoceles. South Med J 1984;77:283-7.

20. Klemi PJ, Nevalainen TJ, Aho AJ. The histogenesis of mucinous cystadenoma of the appendix. Arch Pathol Lab Med
1980;104:162-3.

21. Gibbs NM. Mucinous cystadenoma and cystadenocarcinoma of the vermiform appendix with particular reference to mucocele and pseudomyxoma peritonei. J Clin Pathol 1973;26:413-21.

22. Carr NJ, McCarthy WE, Sobin LE. Epithelial noncarcinoid tumors and tumor-like lesions of the appendix: a clinicopathologic study of 184 patients with a multivariate analysis of prognostic factors. Cancer 1995;75:757-68.

\section{Book Review}

\section{Vigorita VJ: Orthopaedic Pathology, 718 pp, Philadelphia, Lippincott Williams \& Wilkins, 1999 (\$229).}

This is an attractively designed book intended to provide a comprehensive review of orthopaedic diseases. It provides pathologic aspects and clinical-radiologic correlations for anyone who has a serious interest in orthopaedic pathology.

The book is divided into 18 chapters and includes seven appendices that discuss in tabular or abbreviated form ultrastructural findings, flow cytometry, immunohistochemistry, genetic abnormalities, and classification of osteochondrodysplasia. The book is the result of the author's 20 years of dedicated service as an orthopaedic pathologist who has accumulated a lot of experience on this subject not only by studying specimens but also by teaching residents and fellows about the intricate art of orthopaedic pathology. Unlike other books on orthopaedic pathology that are devoted to either bone tumors or other orthopaedic disorders, this book covers both of these subjects very well. The chapters are well illustrated with easy-tofollow tables, outlines, diagrams of metabolic disease pathways, clinical photographs, and a variety of gross and microscopic illustrations, including ultrastructural features when appropriate. The pathologic illustrations, all in color, are of superb quality. An array of radiologic images complement the book.

The first few chapters discuss basic information about bone development and progression. These chapters are the key to an easier understanding of the rest of the book. Two chapters are dedicated to metabolic bone disease. The first deals with the fundamental principles of the evaluation of metabolic bone disease, discussing in detail the management of the specimen, the mode of fixation, embedding in plastics without decalcification, double labeling, and the interpretation of the volume of osteoid and surface extent. Osteoporosis, osteomalacia, and hypophosphatasia are included in this chapter. The second chapter on metabolic bone disease discusses renal osteodystrophy, diseases that induce bone remodeling, collagen-related metabolic bone disease, vitaminosis, and other metabolic bone disorders, complementing the preceding chapter. For a pathologist who seldom deals with metabolic bone disease, these chapters are an eye opener as one finds significant detail to understand the disease processes along with the pathologic interpretation of the bone biopsy. An inexperienced pathologist in metabolic bone disease will find answers for how to proceed with the handling of a specimen of a patient who might have a metabolic bone disease.

The bone tumors are discussed with simplicity, yet are detailed when needed. A plethora of new data coming from molecular investigation is fairly well reflected in this book. However, cytopathology is not included. Treatment is only briefly discussed, as this is not the thrust of the book.

The book is nicely complemented with a chapter on implant pathology, which discusses the types of material used in orthopaedic surgery and the secondary changes that these devices induce in the tissues.

The author has chosen to give some information in chapters preceding the major discussion on certain diseases. For instance, cysts related to arthritis are described in the chapter dedicated to cysts, when they probably should have been discussed within the subject of degenerative osteoarthritis, where they most commonly occur.

Overall, this book is clear, relatively concise, and extremely well illustrated. I highly recommend it for any student of orthopaedic pathology. This book should be on the shelf of any surgical pathologist who may occasionally have to deal with a case of metabolic bone disease or with a primary bone tumor. The book is certainly recommended for orthopaedic residents in training and for fellows in either orthopaedic surgery or bone pathology.

\author{
Alberto G. Ayala \\ M.D. Anderson Cancer Center \\ Houston, Texas
}

